\title{
Bias driven coherent carrier dynamics in a two-dimensional aperiodic potential
}

\author{
F. A. B. F. de Moura \\ Instituto de Física, Universidade Federal de Alagoas, Maceió AL 57072-970, Brazil \\ L. P. Viana \\ Pólo Penedo, Universidade Federal de Alagoas, Penedo AL 57200-000, Brazil \\ M. L. Lyra \\ Instituto de Física, Universidade Federal de Alagoas, Maceió AL 57072-970, Brazil \\ V. A. Malyshev \\ Centre for Theoretical Physis and Zernike Institute for Advanced Materials, \\ University of Groningen, Nijenborgh 4, 9747 AG Groningen, The Netherlands \\ F. Domínguez-Adame \\ GISC, Departamento de Física de Materiales, Universidad Complutense, E-28040 \\ Madrid, Spain
}

\begin{abstract}
We study the dynamics of an electron wave-packet in a two-dimensional square lattice with an aperiodic site potential in the presence of an external uniform electric field. The aperiodicity is described by $\epsilon_{\mathbf{m}}=V \cos \left(\pi \alpha m_{x}^{\nu_{x}}\right) \cos \left(\pi \alpha m_{y}^{\nu_{y}}\right)$ at lattice sites $\left(m_{x}, m_{y}\right)$, with $\pi \alpha$ being a rational number, and $\nu_{x}$ and $\nu_{y}$ tunable parameters, controlling the aperiodicity. Using an exact diagonalization procedure and a finite-size scaling analysis, we show that in the weakly aperiodic regime $\left(\nu_{x}, \nu_{y}<1\right)$, a phase of extended states emerges in the center of the band at zero field giving support to a macroscopic conductivity in the thermodynamic limit. Turning on the field gives rise to Bloch oscillations of the electron wave-packet. The spectral density of these oscillations may display a double peak structure signaling the spatial anisotropy of the potential landscape. The frequency of the oscillations can be understood using a semi-classical approach.
\end{abstract}

Key words: aperiodic potential, coherent electron dynamics, Bloch oscillations 


\section{Introduction}

Materials with restricted geometry, such as semiconductor quantum-well structures, [1] quantum dots and wires, [2,3] organic thin films [4] as well as quasiperiodic structures, [5] are nowadays subjects of growing interest from both fundamental and practical points of view. An attributive peculiarity of almost all of them is the presence of disorder, which can be both of an intrinsic nature (imperfections of the structure itself) and originated from a random environment.

Whenever disorder is involved, Anderson's ideas about localization of quasiparticles states come into play. [6] In three dimensions (3D), the states at the center of the quasiparticle band remain extended for a relatively weak disorder (of magnitude smaller than the bandwidth), while the other states (in the neighborhood of the band edges) turn out to be exponentially localized. This implies the existence of two mobility edges which separates the phases of extended and localized states. [7] On the contrary, uncorrelated disorder of any magnitude causes localization of all one-particle eigenstates in one dimension (1D) [8] and two dimensions (2D). [9]

Since late eighties, however, it has been realized that extended states may survive on $1 \mathrm{D}$ systems if the disorder distribution is correlated. $[10,11,12,13,14,17,15,16,18]$ Thus, a short-range correlated disorder was found to stabilize the extended states at special resonance energies. In the thermodynamic limit, such extended states form a set of null measure in the density of states, $[10,11,12,13,14]$ implying the absence of mobility edges in these systems. In contrast, systems with long-range correlations of disorder support a set of delocalized states within a finite bandwidth, $[15,16]$ giving rise to mobility edges. Theoretical predictions of localization suppression on 1D geometries, due to correlations of the disorder distribution, were confirmed experimentally in semiconductor superlattices with intentional correlated disorder, [17] as well as in single-mode waveguides with correlated scatterers. [18]

Among 1D models with extended states, aperiodic Anderson models [19] with an incommensurate site potential represent a class of particular interest. These models have been extensively investigated in the literature, [20,21,22,19,23] and the localized or extended nature of the eigenstates has been related to

1 On leave from V. A. Fock Institute of Physics, St. Petersburg University, 198904 St.-Petersburg, Russia 
general characteristics of aperiodic site-energy distributions. Interesting realizations of this kind of distribution are achieved when weakly aperiodic potentials are considered: these systems support the occurrence of mobility edges, in a close analogy with the standard 3D Anderson model. [19]

A key question in any general theory of aperiodic systems is the relationship between a given aperiodic site-energy distribution and physical properties of the system. Nowadays, the solution of this problem is still lacking. [24] Nevertheless, some important results have been obtained concerning the dynamics of elementary excitations, like electrons, phonons, excitons, polaritons, spin waves, plasmons or magnons, propagating through different classes of aperiodic lattices. $[25,5,26,27]$ In these studies, relevant physical properties were analyzed in terms of appropriate model Hamiltonians.

Recently, the dynamics of a single electron in homogeneous systems subjected to a uniform static electric field has received much attention. Under this constraint, the electron undergoes the so-called Bloch oscillations, [28,29,30,31,32] whose amplitude is proportional to the energy bandwidth. Electronic Bloch oscillations were observed for the first time in semiconductor superlattices [33] (see Ref. [34] for an overview). A similar phenomenon of sustained oscillations of the electromagnetic field, named photon Bloch oscillations, have been also found in 2D waveguide arrays and optical superlattices based on porous silicon. [35] In disordered systems, Bloch oscillations were predicted to occur in both 1D [36] and 2D [37] geometries whenever the disorder distribution is long-range correlated. The amplitude of the oscillations was found to carry information about the energy difference between the two mobility edges, existing in these systems. The occurrence of Bloch oscillations was also theoretically argued for 1D chains with aperiodic slowly varying potentials. [38] A spectroscopic manifestation of Bloch oscillations is found in the appearance of the Wannier-Stark ladder in the absorption spectrum. [39]

In this paper, apart from the generalization of the previous work, [19] done for a one-dimensional system, to a 2D system, we consider an electron subjected to an external uniform electric field. The latter brings new features to the electron wave packet dynamics moving in incommensurate potential. In particular, despite a quasi-stochasticy of the system, sustained Bloch oscillations can be observed, previously believed to exist only for a periodic structures. In order to produce an aperiodic (2D) site potential, the formalism used in Ref. [19] was considered. It consists in using a sinusoidal function whose phases $\phi_{x}$ and $\phi_{y}$ varies as a power-law. The power-law exponent controls the degree of aperiodicity in the site potential. We start by employing an exact diagonalization formalism to compute the $d c$ conductance and participation number in the absence of the electric field. We numerically demonstrate that their size behavior is consistent with the existence of extended states near the band center for weakly aperiodic potentials. In this regime, the wave-packet dynamics 
becomes ballistic. Furthermore, we focus on the wave-packet dynamics in the presence of an external uniform electric field. We show that in the limit of a weakly aperiodic site potential, the electric field promotes sustained Bloch oscillations of the electronic wave-packet. The frequency of these oscillations can be understood within the framework of a semi-classical approach.

The outline of the paper is as follows. In the next section we present our model and quantities of interest. In Sec. 3, the results of the numerical simulations of the dc conductance, participation number and electronic wave-packet dynamics are discussed. We summarize in Sec. 4.

\section{Model and formalism}

We consider a tight-binding single electron Hamiltonian on a regular 2D open lattice of spacing $a$ with an aperiodic site potential and a uniform static electric field $[32,40]$

$$
\begin{aligned}
H & =\sum_{\boldsymbol{m}}\left(\epsilon_{\boldsymbol{m}}+\boldsymbol{U} \cdot \boldsymbol{m}\right)|\boldsymbol{m}\rangle\langle\boldsymbol{m}| \\
& +J \sum_{\langle\boldsymbol{m} \boldsymbol{n}\rangle}(|\boldsymbol{m}\rangle\langle\boldsymbol{n}|+| \boldsymbol{n}\rangle\langle\boldsymbol{m}|),
\end{aligned}
$$

where $|\boldsymbol{m}\rangle$ is a Wannier state localized at site $\boldsymbol{m}=m_{x} \boldsymbol{e}_{x}+m_{y} \boldsymbol{e}_{y}, \epsilon_{\boldsymbol{m}}$ is its energy, and $\boldsymbol{U}=e \boldsymbol{F} a$ is the energetic bias given by the electric field $\boldsymbol{F},-e$ being the electron charge. Here $\boldsymbol{e}_{x}$ and $\boldsymbol{e}_{y}$ are the corresponding Cartesian unit vectors. We will assume that the electric field $\boldsymbol{F}$ is applied along the diagonal of the square lattice, i.e., $\boldsymbol{U}=U\left(\boldsymbol{e}_{x}+\boldsymbol{e}_{y}\right) / \sqrt{2}$, were $U$ is the bias. Transfer integrals are restricted to the nearest-neighbor interaction and are given by $J$. Hereafter, the energy scale is fixed by setting $J=1$.

The 2D aperiodic potential $\epsilon_{\boldsymbol{m}}$ is taken in the form:

$$
\epsilon_{\boldsymbol{m}}=V \cos \left(\pi \alpha m_{x}^{\nu_{x}}\right) \cos \left(\pi \alpha m_{y}^{\nu_{y}}\right)
$$

where $V, \alpha, \nu_{x}$, and $\nu_{y}$ are variable parameters.

Equation (2) is a 2D generalization of the aperiodic 1D potential introduced by Das Sarma et al. [19] In $1 D$ and at $\nu=1$, this potential represents just Harper's model. In this case, a rational $\alpha$ describes a crystalline solid, whereas an irrational $\alpha$ yields an incommensurate potential. It was shown in Ref. [21] that for $\nu>1$ all one-electron states are localized. In this regime, the frequency

of the potential oscillations diverge in the thermodynamic limit, resulting in a 
fairly uncorrelated sequence of the on-site energies. Because of that, this case is usually named as the pseudo-random regime. Oppositely, it was demonstrated that the range $0<\nu<1$ supports a phase of extended states in the neighborhood of the band center. [19] In contrast to the pseudo-random regime, it is the average wavelength of the potential oscillations that diverges in the thermodynamic limit. Because of the slower divergence of the oscillation wavelength, this regime is usually named as weakly aperiodic. The uniform case is recovered at $\nu=0$. Recently, the effect of this kind of aperiodicity on the phonon and magnon $1 D$ modes has attracted a renewed interest. [26,27]

The time-dependent Schrödinger equation governs the dynamics of the Wannier amplitudes, [41]

$$
\begin{aligned}
i \dot{\psi}_{\boldsymbol{m}} & =\left(\epsilon_{\boldsymbol{m}}+\boldsymbol{U} \cdot \boldsymbol{m}\right) \psi_{\boldsymbol{m}} \\
& +\left(\psi_{\boldsymbol{m}+\boldsymbol{e}_{x}}+\psi_{\boldsymbol{m}-\boldsymbol{e}_{x}}+\psi_{\boldsymbol{m}+\boldsymbol{e}_{y}}+\psi_{\boldsymbol{m}-\boldsymbol{e}_{y}}\right),
\end{aligned}
$$

where the Planck constant $\hbar$ is set to unity.

We solve numerically Eq. (3) to study the time evolution of an initially Gaussian wave-packet centered at site $\boldsymbol{m}_{0}$,

$$
\psi_{\boldsymbol{m}}(t=0)=A \exp \left[-\frac{\left(\boldsymbol{m}-\boldsymbol{m}_{0}\right)^{2}}{4 \Delta^{2}}\right],
$$

where $A$ is the normalization constant and we set $\Delta=1$ hereafter. Once Eq. (3) is solved for the initial condition (4), we compute the projection of the mean position of the wave-packet (centroid) and the average velocity $v(t)$ along the field direction

$$
\begin{aligned}
R(t) & =\frac{1}{\sqrt{2}}\left[m_{x}(t)+m_{y}(t)\right], \\
v(t) & =-2 \sum_{\boldsymbol{m}} \psi_{\boldsymbol{m}}\left[\psi_{\boldsymbol{m}+\boldsymbol{e}_{x}}^{*}+\psi_{\boldsymbol{m}+\boldsymbol{e}_{y}}^{*}\right],
\end{aligned}
$$

as well as the spread of the wave-function (square root of the mean-square displacement)

$$
\sigma(t)=\sqrt{\sum_{\boldsymbol{m}}[\boldsymbol{m}-\boldsymbol{m}(t)]^{2}\left|\psi_{\boldsymbol{m}}(t)\right|^{2}}
$$

where $\boldsymbol{m}(t)=\sum_{\boldsymbol{m}} \boldsymbol{m}\left|\psi_{\boldsymbol{m}}(t)\right|^{2}$.

In addition, we use the exact diagonalization of the Hamiltonian (1) in the absence of the electric field $(U=0)$ to obtain the eigenvalues and eigenvectors, and to calculate the $d c$ conductance $G(E)$ as a function of energy $E$. For 
noninteracting electrons and within the linear response theory at $T=0 \mathrm{~K}$, $G(E)$ is given by the Kubo-Greenwood formula [42] (in units of $e^{2} / \hbar$ )

$$
\begin{aligned}
G(E) & =\frac{2 \pi}{\Omega} \sum_{a, b}|\langle a|x H-H x| b\rangle|^{2} \\
& \times \delta\left(E-E_{a}\right) \delta\left(E-E_{b}\right) .
\end{aligned}
$$

Here, the polarization is taken in the direction of the $x$-axis, $\Omega$ is the area of the system, $x$ is the position operator, and the indices $a$ and $b$ label the eigenstates. We also compute the participation number of a normalized eigenstate as follows

$$
P(E)=\left[\sum_{\boldsymbol{m}}\left|\psi_{\boldsymbol{m}}(E)\right|^{4}\right]^{-1}
$$

For extended states, $P(E)$ is expected to scale linearly with the number of sites. More specifically, we will focus on the $d c$ conductance and the participation number near the band center, averaged over a narrow energy stripe at the band center $\left[-W_{c} / 2, W_{c} / 2\right]$ :

$$
\begin{aligned}
& G=\frac{1}{N_{E}} \sum_{|E|<W_{c} / 2} G(E), \\
& \xi=\frac{1}{N_{E}} \sum_{|E|<W_{c} / 2} P(E),
\end{aligned}
$$

where $N_{E}$ is the number of eigenstates within the stripe.

\section{Results and Discussion}

In all our numerical simulations, we set the parameters $V$ and $\pi \alpha$ of the aperiodic potential to $V=3$ and $\pi \alpha=1$, while considering the exponents $\nu_{x}$ and $\nu_{y}$ as variables. When calculating $G(E)$, Eq. (7), the $\delta$-functions were replaced by $\delta(E)=(1 / \Delta E) \Theta(\Delta E / 2-|E|)$, with $\Delta E=0.1, \Theta$ being the Heaviside step-function. The magnitude of the energy stripe in Eqs. (9a) and (9b) was chosen $W_{c}=0.2$.

\subsection{Zero-field electronic states}

Firstly, we discuss the character of the electronic states of the model under study. Figure 1(a) displays the averaged conductance $G$ at the band center $(E=0)$ as a function of $\nu \equiv \nu_{x}=\nu_{y}$, obtained after numerical diagonalization of the Hamiltonian (1) in the absence of the external electric field $(U=0)$. To 
avoid strong fluctuations of the conductance related to particular realizations of the aperiodic potential, we further averaged the results over a narrow range of potential amplitudes $(\Delta V=0.05)$. As we observe from Fig. 1(a), for a slowly varying aperiodic site potential $\left(\nu_{x}, \nu_{y}<1\right)$ the conductance grows on increasing the system size. This is a signature of macroscopic conductance in the thermodynamic limit. In contrast, the conductance is almost null for a pseudo-random potential $\left(\nu_{x}, \nu_{y}>1\right)$, in agreement with previous calculations for localized eigenmodes. [42] A similar trend is found in the behavior of the averaged participation number $\xi$, as shown in Fig. 1(b). For weakly aperiodic potentials, $\xi$ grows with the system size, while it becomes size independent for strongly aperiodic (pseudo-random) systems.
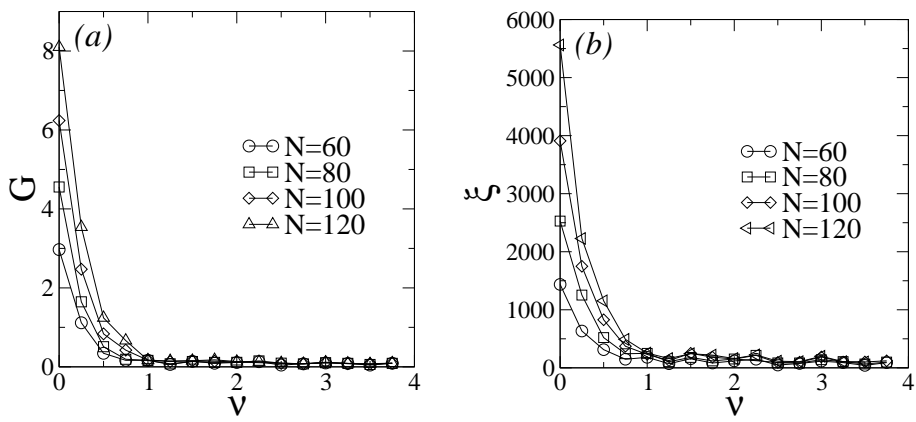

Fig. 1. (a) Averaged $d c$ conductance $G$, Eq. (9a), and (b) averaged participation number $\xi$, Eq.(9b), versus the exponent $\nu=\nu_{x}=\nu_{y}$ calculated for different system sizes $N$. The average was performed considering a narrow range of potential amplitudes $(\Delta V=0.05)$ to reduce statistical fluctuations.

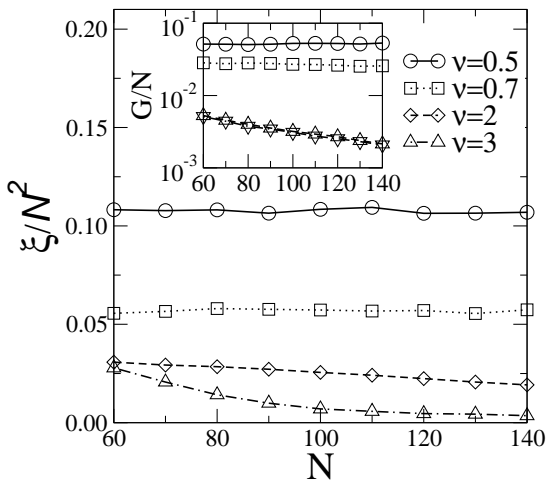

Fig. 2. Finite size scaling of the normalized participation number $\xi / N^{2}$ for the states in the vicinity of the band center calculated for various values of the exponent $\nu$ (shown in the legend). The inset presents the finite-size scaling of the normalized conductance $G / N$. The different character of the finite-size scaling of both quantities on increasing the exponent $\nu$ signals the change in the nature of electronic states in the band center from delocalized $(\nu<1)$ to localized ones $(\nu>1)$.

The correlations found in the behavior of the averaged conductance $G$ and the participation number $\xi$ as a function of the exponent $\nu$ indicate that the model under study undergoes a metal-insulator transition associated with a change in the nature of the one-electron eigenstates. To give further support to this 
finding, we performed a finite-size scaling analysis of these two quantities. In Fig. 2 we plotted the size dependence of the normalized participation number $\xi / N^{2}$ for various values of the exponent $\nu$. As it can be noticed, $\xi / N^{2}$ does not depend of $N$ in the regime of weak aperiodicity $(\nu<1)$, i.e., the participation number itself $\xi$ is proportional to the total number of sites $N^{2}$. This is a clear indication that the eigenstates at the band center are delocalized over a finite fraction of the lattice. On the contrary, for strong aperiodic potentials $(\nu>1)$, $\xi / N^{2}$ decreases with the system size, signaling the wave-function localization. The size dependence of the normalized conductance $G / N$ in the center of the band, shown in the inset of Fig. 2, corroborates the conclusion above. In the slowly varying aperiodic regime $G \propto N$ leading to a macroscopic transport supported by extended states. Such a behavior is expected for a regular square lattice, where the conductance at the band center should be proportional to the number of conducting channels, i.e., to the linear size of the lattice $N$. In the limit of strongly aperiodic lattices, the normalized conductance decreases with the system size, thus indicating the absence of macroscopic transport in the thermodynamic limit. This is in agreement with the standard scaling theory of Anderson localization. [9]

\subsection{Dynamics}

We also study the time evolution of a wave-packet initially localized in the center of the lattice, $\mathbf{m}_{0}=(N / 2, N / 2)$, to uncover the character of the electronic wave-packet dynamics in the present model system. We start our analysis analyzing the wave-packet dynamics in the absence of the external field $(U=0)$. We numerically integrate the wave-equation (3) and calculate the wave-packet spread $\sigma(t)$ until it reaches a stationary value as a result of multiple reflections of the electron on the lattice boundaries. The extended states that appear in this model emerge in the regime at which the potential has a diverging wavelength in the thermodynamic limit. These are typically non scattered modes thus leading to a ballistic wave-packet spread, $\sigma(t) \propto t$. This means that, as long as $\sigma(t) \leq N$, the data points calculated for different system sizes $N$ should collapse into a unique straight line after re-scaling $\sigma(t) \rightarrow \sigma(t) / N$ and $t \rightarrow t / N$. In the localized regime, such a collapse is not expected. We further calculated the time-dependent participation second moment $\xi(t)=1 / \sum_{\boldsymbol{m}}\left|\psi_{\boldsymbol{m}}(t)\right|^{4}$. In general, $\xi(t)$ scales linearly with the number of sites whenever the system supports extended states.

In Fig. 3, we plot the results of our calculations. For a weakly aperiodic site potential $\left(\nu_{x}=\nu_{y}=0.5\right)$, we found the above mentioned collapse of all curves into a unique straight line for $\sigma(t) \leq N$ [see Fig. 3(a)]. This allow us to conclude that the wave-packet motion in this case has a ballistic nature, signaling the presence of a phase of delocalized non scattered states. In the case of a 
pseudo-random site potential $\left(\nu_{x}=\nu_{y}=1.5\right)$, such a collapse is seen only for $\sigma(t) \ll N$ [see Fig. 3(b)]: the wave-packet motion is ballistic whenever $\sigma(t)$ is smaller than the largest localization length. For long times, a collapse is absent [see Fig. 3(b)], indicating the wave-packet localization. In Fig. 3(c), we show results for the time-dependent participation second moment for $\nu_{x}=\nu_{y}=0.5$ (solid line) and $\nu_{x}=\nu_{y}=1.5$ (dashed line). For $\nu<1$, the participation dynamics show a ballistic behavior until the wave-packet reaches the lattice boundaries. For $\nu>1$ a slower dynamics was obtained. These results are in perfect agreement with the wave-packet spread calculations, indicating the presence of non scattered modes in the initial wave-packet only in the regime of weakly aperiodic potentials $(\nu<1)$. These data corroborate our statement about the Anderson transition for the underlined 2D aperiodic model, which we claimed in the previous section on the basis of the finite size scaling of the conductance $G$ and participation number $\xi$ for the states in the vicinity of the band center.
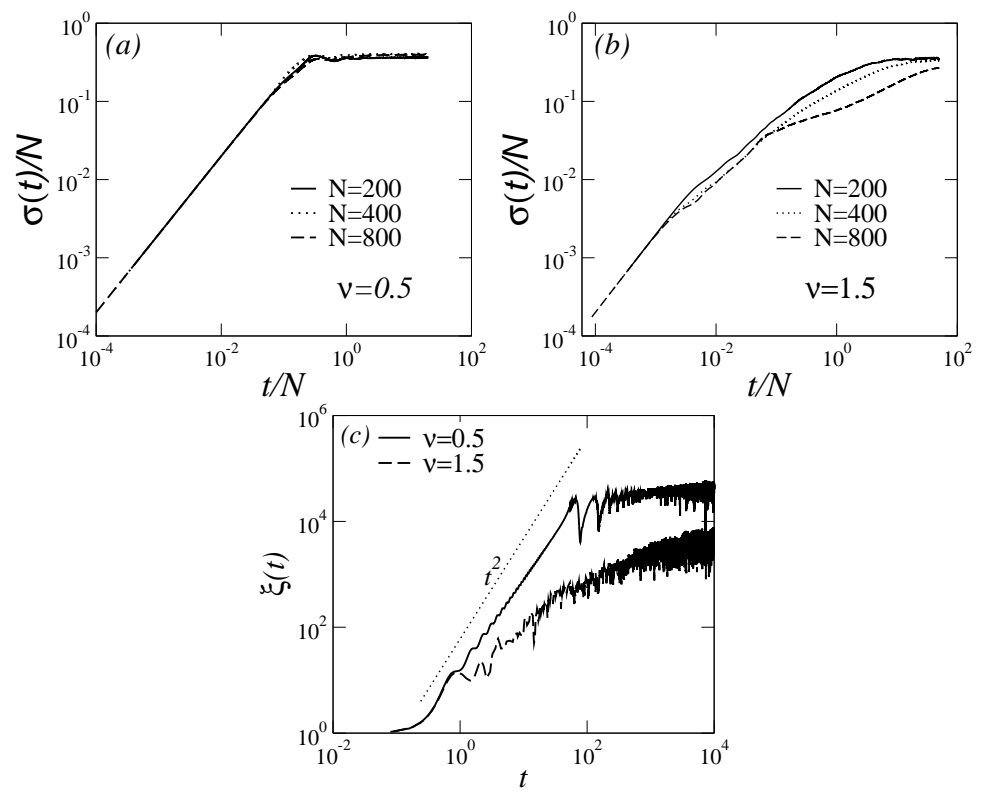

Fig. 3. The normalized wave-packet spread $\sigma(t) / N$, Eq. (6), as a function of the re-scaled time $t / N$ computed for lattices of $N \times N=200 \times 200$ to $800 \times 800$ sites at zero bias $(U=0)$. (a) A slowly varying (weakly aperiodic) site potential $\left(\nu_{x}=\nu_{y}=\nu=0.5\right)$. The collapse of all curves into a unique curve until $\sigma(t) \leq N$ indicates the presence of non scattered extended states. (b) A pseudo-random potential $\left(\nu_{x}=\nu_{y}=\nu=1.5\right)$. A similar collapse is absent, signalling localization. (c) Time dependent participation $\xi(t)$ number versus time $t$ computed for $N \times N=1600 \times 1600$ and $\nu_{x}=\nu_{y}=\nu=0.5$ (solid line) and $\nu_{x}=\nu_{y}=\nu=1.5$ (dashed line). In agreement with the wave-packet spread calculations, the participation number spreads ballistically $\left(\xi(t) \propto t^{2}\right)$ for $\nu<1$ indicating the presence of non scattered modes.

Now, we turn to the wave-packet dynamics of an electron subjected to a uniform electric field $(U \neq 0)$. It is well known that in disorder-free systems, 

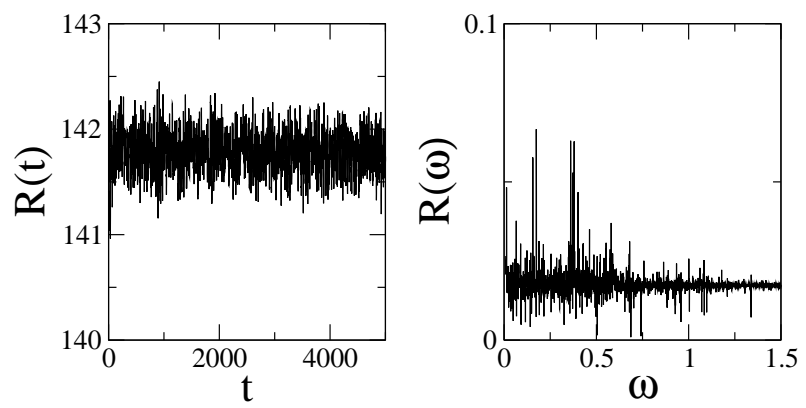

Fig. 4. Bias driven dynamics of the centroid $R(t)$, Eq. (5), in a pseudo-random potential $\left(\nu_{x}=\nu_{y}=1.5\right)$ with $N \times N=200 \times 200$ sites and bias magnitude $U=0.5$. No signature of coherent Bloch oscillations is seen in this case. The Fourier transform $\tilde{R}(\omega)$ of the centroid $R(t)$ is broad, supporting the absence of a typical oscillation frequency in $R(t)$.
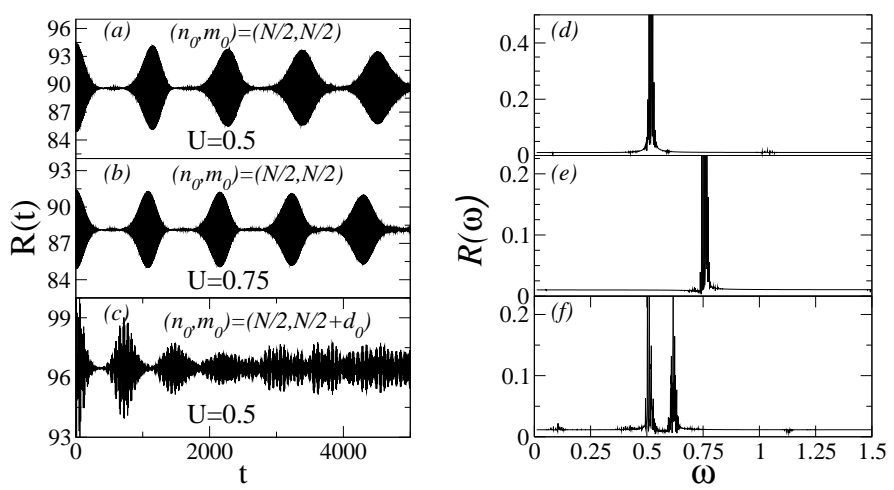

Fig. 5. Bias driven dynamics of the centroid $R(t)$ in a slowly varying (weakly aperiodic) potential $\left(\nu_{x}=\nu_{y}=0.5\right)$ for two magnitudes of the bias: (a) $U=0.5$ and (b) $U=0.75$. The initial location of the wave-packet is $\left(n_{0}, m_{0}\right)=(N / 2, N / 2)$. (d) and (e) Fourier transforms $\tilde{R}(\omega)$ of the centroids depicted on panels (a) and (b), respectively. Note that $\tilde{R}(\omega)$ is peaked at a frequency $\omega \simeq U$. (c) Same as in panel (a), but for the initial condition $\left(n_{0}, m_{0}\right)=\left(N / 2, N / 2+d_{0}\right)$ with $d_{0}=10$. (f) Fourier transform of the centroid depicted on panel (c). Note that here $\tilde{R}(\omega)$ exhibits a doublet structure.

a uniform electric field causes dynamic localization of the electron and gives rise to an oscillatory motion of the wave-packet, the so-called Bloch oscillations. [28,29] The size of the segment over which the electron oscillates and the period of the oscillations are estimated from semiclassical arguments to be $L_{F}=a W / U$ and $\tau_{B}=2 \pi / U$, respectively, [43] where $W$ is the width of the Bloch band.

Firstly, we compute the wave-packet centroid $R(t)$ in a pseudo-random potential $\left(\nu_{x}=\nu_{y}=1.5\right)$ of size $N \times N=200 \times 200$ with the bias magnitude $U=0.5$. As deduced from Fig. 4, there is no signature of Bloch oscillations in this case. Coherent oscillations, which are present immediately after the initial wave-packet is released, are quickly destroyed (not shown). The asymptotic behavior of the centroid resembles a stochastic motion around some mean po- 


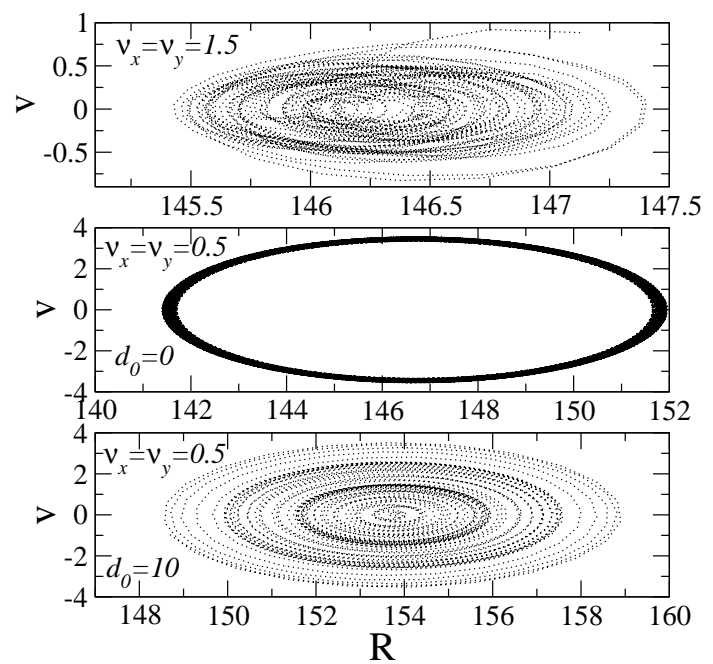

Fig. 6. Phase diagrams velocity-vs-position computed for: (a) $\nu_{x}=\nu_{y}=1.5$, $U=0.5$ and the initial location of the wave-packet $\left(n_{0}, m_{0}\right)=(N / 2, N / 2)$; (b) $\nu_{x}=\nu_{y}=0.5, U=0.5$ and the initial location of the wave-packet $\left(n_{0}, m_{0}\right)=(N / 2, N / 2)$; (c) the same as in (b) except the initial location of the wave-packet $\left(n_{0}, m_{0}\right)=\left(N / 2, N / 2+d_{0}\right)$ with $d_{0}=10$. For $\nu>1$ the velocity-vsposition diagram exhibits incoherent orbits. For $\nu<1$, the Bloch-like oscillations promotes a coherent dynamics through the phase space. In panel (c), the amplitude of the oscillations varies faster than in panel (b) due to the doublet structure generated by the anisotropic gradient of the local potential.

sition. The Fourier spectrum $\tilde{R}(\omega)$ of the centroid, plotted in the right panel of Fig. (4), confirms this claim. The spectrum is rather broad, with no characteristic frequencies, indicating that $R(t)$ is similar to a white noise signal. Thus, for $\nu_{x}, \nu_{y}>1$ the system shows a behavior similar to the standard Anderson model, with no signature of coherent Bloch oscillations.

In the case of a weakly aperiodic potential $\left(\nu_{x}=\nu_{y}=0.5\right)$, the centroid reveals an oscillatory amplitude-modulated pattern, as seen in Fig. 5 (panels a, b, and c). Its Fourier transform $\tilde{R}(\omega)$ displays a well-defined narrow peak, the location of which is slightly deviated from the expected value $\omega=U$ (panels $\mathrm{d}$ and e); this small shift is caused by the local contribution to the external bias produced by the modulated potential. Indeed, the bias at position $\left(n_{0}, m_{0}\right)$ is given by $\boldsymbol{U}_{\text {eff }}=\left(\delta \varepsilon_{n, m} / \delta n+U, \delta \varepsilon_{n, m} / \delta m+U\right)$. The local contribution shall be relevant whenever the potential gradient (in appropriate units) is of the order of the external bias $U$. Therefore, the frequency obtained in not exactly $\omega=U$ [Figs. 5(a) and 5(b)]. The two peak structure depicted in Fig. 5(c) has its origin in the distinct contributions given by the anisotropic gradient of the local potential to the effective local bias. Once the initial wave-packet is located at a non-symmetrical position of the lattice $\left(n_{0}, m_{0}\right)=\left(N / 2, N / 2+d_{0}\right)$ with $d_{0}=$ 10 , the potential derivatives along the orthogonal lattice directions give raise to distinct frequency shifts when the electron is forced by a diagonal external field. We stress that such frequency splitting is not found in $1 D$ aperiodic 

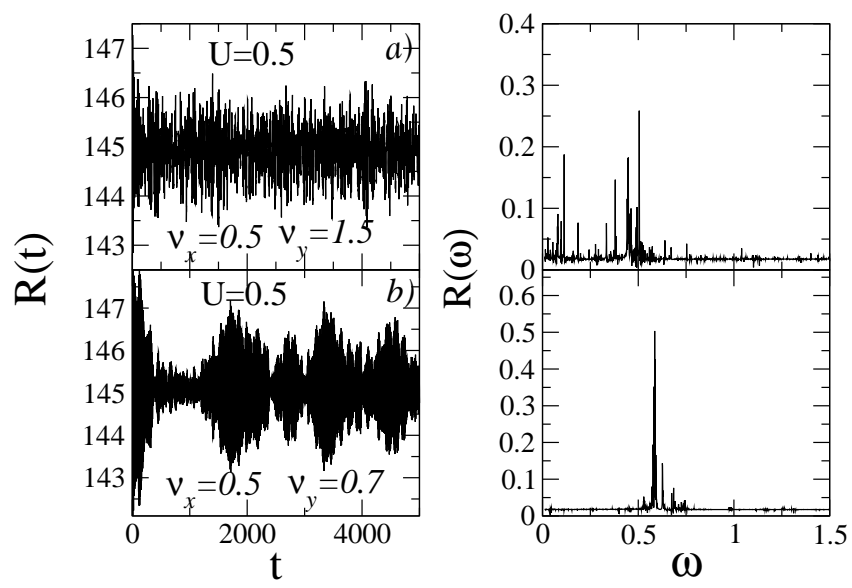

Fig. 7. The dynamics of the centroid $R(t)$ and its Fourier transform (left and right panel respectively) for $\nu_{x}=0.5$ and (a) $\nu_{y}=1.5$ and (b) $\nu_{y}=0.7$. For both $\nu_{x}$ and $\nu_{y}$ below 1, Bloch-oscillation with a frequency close to the one predicted by the semi-classical approach was obtained. However, in the case of mixed pseudo-random and weakly aperiodic components, the pseudo-random character for $\nu_{y}>1$ predominates leading to the Anderson localization of the one-electron eigenstates and the consequent incoherent dynamics of the wave-packet centroid.

potentials, being thus a specific feature of higher dimensional systems.

In Fig. 6, we present the phase space plots, namely velocity versus position . All calculations were performed using $N \times N=200 \times 200$. Three representative cases are illustrated: (a) $\nu_{x}=\nu_{y}=1.5, U=0.5$ with the initial location of the wave-packet $\left(n_{0}, m_{0}\right)=(N / 2, N / 2)$; (b) $\nu_{x}=\nu_{y}=0.5, U=0.5$ with the initial location of the wave-packet $\left(n_{0}, m_{0}\right)=(N / 2, N / 2)$; (c) the same as in (b) except by initial location of the wave-packet $\left(n_{0}, m_{0}\right)=\left(N / 2, N / 2+d_{0}\right)$ with $d_{0}=10$. For all cases, the integration time used was $t=1000$. We can see in (a) that the velocity versus position diagram exhibits orbits with no coherency, as it was observed in the centroid dynamics (see Fig. 4). In the other cases, the diagrams show a coherent dynamics through the phase space. In the case of bimodal Bloch oscillations [see Fig. 6(c)], the wave-packet orbits in phase space show a pronounced breathing pattern. This breathing reflects the fact that both centroid and velocity display amplitude-modulated envelopes. The frequency of this amplitude-modulated envelope depends on the difference between the two frequency peaks appearing the Bloch oscillations spectrum [Fig. 5(c)].

Before concluding, some words concerning the electronic dynamics in systems with distinct degrees of aperiodicity along the orthogonal lattice directions $\nu_{x} \neq \nu_{y}$. In Fig.7, we show the centroid $R(t)$ and its Fourier transform (left and right panel respectively) for an initial Gaussian wave-packet located at the center of the lattice with aperiodicity exponents $\nu_{x}=0.5$ and (a) $\nu_{y}=1.5$ and (b) $\nu_{y}=0.7$. For both $\nu_{x}$ and $\nu_{y}$ below 1 , we can see oscillations with a frequency close to the Bloch-oscillation frequency predicted by the semi- 
classical approach. However, in the case of mixed pseudo-random and weakly aperiodic components, the pseudo-random character for $\nu_{y}>1$ predominates leading to the Anderson localization of the one-electron eigenstates and the consequent incoherent dynamics of the wave-packet centroid.

\section{Summary and concluding remarks}

In this work, we studied numerically the dynamics of a single electron wavepacket moving in a 2D square lattice with an aperiodic site potential $\epsilon_{m}=$ $V \cos \left(\pi \alpha m_{x}^{\nu_{x}}\right) \cos \left(\pi \alpha m_{y}^{\nu_{y}}\right)$ in the presence of an external uniform electric field. For fixed parameters $V$ and $\pi \alpha$, the exponents $\nu_{x}$ and $\nu_{y}$ allow to control the degree of aperiodicity.

We have discussed the character of the electronic states of the model in the absence of an external electric field. Diagonalizing numerically the Hamiltonian at zero electric field, we computed the $d c$ conductance and the participation number for various systems sizes. After a finite-size scaling analysis of data close to the band center, we found that, for a weakly aperiodic potential $\left(\nu_{x}, \nu_{y}<1\right)$, both quantities grow on increasing the system size. The participation number is proportional to $N^{2}$ while the conductance scales proportional to $N$. Such size dependencies are consistent with the presence of extended states and macroscopic transport in the thermodynamic limit. In contrast, both participation number and conductance reach a plateau upon increasing the size for a pseudo-random potential $\left(\nu_{x}, \nu_{y}>1\right)$, which is the typical behavior predicted by the usual scaling theory of Anderson localization. Solving numerically the time-dependent Schrödinger equation, we found that an initially localized wave-packet reveals a ballistic spreading associated with the existence of a phase of extended non scattered states in the weakly aperiodic limit $\left(\nu_{x}, \nu_{y}<1\right)$. In contrast, the spread of the wave-packet is bounded for pseudo-random realization of the site potential $\left(\nu_{x}, \nu_{y}>1\right)$. These results indicate that the underlined $2 D$ aperiodic model under study undergoes a metal-insulator transition.

We have also investigated the interplay between the delocalization effect, preserved by the weakly aperiodic structure, and the dynamic localization, caused by an electric field acting on the system. We demonstrated that in weakly

aperiodic potentials $\left(\nu_{x}, \nu_{y}<1\right)$, the applied uniform electric field promotes sustained Bloch oscillations of the electronic wave-packet. The frequency of the oscillations was shown to agree with the prediction of a semi-classical approach. [43] In contrast with the Bloch oscillations in $1 D$, the frequency spectrum of the centroid time-evolution can exhibit a two-mode structure depending on the initial position of the wave-packet. Such two-frequency dynamics reflects the anisotropy of the potential landscape along the principal 
lattice directions when the electron is forced to oscillate by a diagonal field. In phase-space, this phenomenon is signaled by quickly breathing coherent orbits.

\section{Acknowledgments}

Work at Alagoas was supported by CNPq-Rede Nanobioestruturas, CAPES (Brazilian research agencies) and FAPEAL (Alagoas State agency). Work at Madrid was supported by MEC (Project MOSAICO) and BSCH-UCM (Project PR34/07-15916).

\section{References}

[1] G. Khitrova, H. M. Gibbs, F. Jahnke, M. Kira, and S. W. Koch, Rev. Mod. Phys. 71, 1591 (1999).

[2] See contributions to Optics of Quantum Dots and Wires, eds. G. W. Briant and G. S. Solomon (Boston: Artech House, 2005).

[3] T. Brandes, Phys. Rep. 408, 315 (2005).

[4] J. R. Tischler, M. S. Bradly, Q. Zhang, T. Atay, A. Nurmiko, and V. Bulović, Org. Electronics 8, 94 (2007).

[5] E. L. Albuquerque and M. G. Cottam, Phys. Rep. 376225 (2003); Polaritons in Periodic and Quasiperiodic Structures (Elsevier: Amsterdam, 2004).

[6] P. W. Anderson, Phys. Rev. 109, 1492 (1958).

[7] N. F. Mott, J. Non-Cryst. Solids 1, 1 (1968).

[8] N. Mott and W. D. Twose, Adv. Phys. 10, 107 (1961).

[9] E. Abrahams, P. W. Anderson, D. C. Licciardello, and T. V. Ramakrishnan, Phys. Rev. Lett. 42, 673 (1979).

[10] J. C. Flores, J. Phys.: Condens. Matter 1, 8471 (1989).

[11] D. H. Dunlap, H.-L. Wu, and P. W. Phillips, Phys. Rev. Lett. 65, 88 (1990).

[12] P. W. Phillips and H.-L. Wu, Science 252, 1805 (1991).

[13] A. Sánchez and F. Domínguez-Adame, J. Phys A:Math. Gen. 27, 3725 (1994); A. Sánchez, E. Maciá, and F. Domínguez-Adame, Phys. Rev. B 49, 147 (1994).

[14] E. Diez, A. Sánchez, F. Domínguez-Adame, Phys Rev. B 50, 14359 (1994); F. Domínguez-Adame, E.Diez, and A. Sánchez, Phys. Rev. B 51, 8115 (1995). 
[15] F. A. B. F. de Moura and M. L. Lyra, Phys. Rev. Lett. 81, 3735 (1998); 84, 199 (2000); Physica A 266, 465 (1999).

[16] F. M. Izrailev and A. A. Krokhin, Phys. Rev. Lett. 82, 4062 (1999).

[17] V. Bellani, E. Diez, R. Hey, L. Toni, L. Tarricone, G. B. Parravicini, F. Domínguez-Adame, and R. Gómez-Alcalá, Phys. Rev. Lett. 82, 2159 (1999).

[18] U. Kuhl, F. M. Izrailev, A. A. Krokhin, and H.-J. Stöckmann, Appl. Phys. Lett. 77, 633 (2000).

[19] S. Das Sarma, S. He, and X. C. Xie, Phys. Rev. Lett. 61, 2144 (1988); Phys. Rev. B 41, 5544 (1990).

[20] D. R. Grempel, S. Fishman, and R. E. Prange, Phys. Rev. Lett. 49, 833 (1982).

[21] M. Griniasty and S. Fishman, Phys. Rev. Lett. 60, 1334 (1988).

[22] D. J. Thouless, Phys. Rev. Lett. 61, 2141 (1988).

[23] H. Yamada, Phys. Rev. B 69, 014205 (2004).

[24] E. Maciá, Rep. Prog. Phys. 69, 397 (2006).

[25] E. Maciá and F. Domínguez-Adame, Electrons, Phonons and Excitons in Low Dimensional Aperiodic Systems, Editorial Complutense, Madrid (2000).

[26] F. A. B. F. de Moura, L. P. Viana, and A. C. Frery, Phys. Rev. B 73, 212302 (2006).

[27] F. A. B. F. de Moura, Eur. Phys. J. B 58, 389 (2007).

[28] F. Bloch, Z. Phys. 52, 555 (1927).

[29] C. Zener, Proc. R. Soc. London, Ser. A 145, 523 (1934).

[30] L. Esaki and R. Tsu, IBM J. Res. Div. 14, 61 (1970).

[31] G. H. Wannier, Phys. Rev. 100, 1227 (1955); Phys. Rev. 101, 1835 (1956); Phys. Rev. 117, 432 (1960); Rev. Mod. Phys. 34, 645 (1962).

[32] D. H. Dunlap and V. M. Kenkre, Phys. Rev. B 34, 3625 (1986).

[33] K. Leo, P. Hairing, F. Brüggemann, R. Schwedler and K. Köhler, Solid State Commun. 84, 943 (1992).

[34] K. Leo, Semicond. Sci. Technol. 13, 249 (1998).

[35] V. Agarwal, J. A. delRio, G. Malpuech, M. Zamfirescu, A. Kavokin, D. Coquillat, D. Scalbert, M. Vladimirova and B. Gil, Phys. Rev. Lett. 92, 097401 (2004).

[36] F. Domínguez-Adame, V. A. Malyshev, F. A. B. F. de Moura, and M. L. Lyra, Phys. Rev. Lett. 91, 197402 (2003). 
[37] F. A. B. F. de Moura, M. L. Lyra, F. Domínguez-Adame, and V. A. Malyshev, J. Phys.: Condens. Matter 19, 056204 (2007).

[38] F. Domínguez-Adame, V. A. Malyshev, F. A. B. F. de Moura, and M. L. Lyra, Phys. Rev. B 71, 104303 (2005).

[39] E. Díaz, F. Domínguez-Adame, Yu. A. Kosevich, and V. A. Malyshev, Phys. Rev. B 73, 174210 (2006).

[40] H. N. Nazareno and P. E. de Brito, Phys. Rev. B 60, 4629 (1999).

[41] B. Kramer and A. MacKinnon, Rep. Prog. Phys. 56 1469, (1993).

[42] M. Tit and M. Schreiber, J. Phys.: Condens. Mattter. 7, 5549 (1995).

[43] N. W. Ashcroft and N. D. Mermin, Solid State Physics (Saunders Colege Publishers, New York, 1976), P. 213. 